\title{
No Neurochemical Evidence of Neuronal Injury or Glial Activation in Children with Paediatric Acute- onset Neuropsychiatric Syndrome
}

Mats Johnson ( $\sim$ mats.johnson@gnc.gu.se)

Goteborgs universitet Sahlgrenska Akademin https://orcid.org/0000-0001-5195-3379

Elisabeth Fernell

Goteborgs universitet Sahlgrenska Akademin

Christopher Gillberg

Goteborgs universitet Sahlgrenska Akademin

Anders Fasth

Goteborgs universitet Sahlgrenska Akademin

Kaj Blennow

Goteborgs universitet Sahlgrenska Akademin

Henrik Zetterberg

Goteborgs universitet Sahlgrenska Akademin

\section{Research}

Keywords: Pediatric Acute-onset Neuropsychiatric Syndrome, PANS, Glial Fibrillary Acidic Protein, GFAP, Neurofilament light, NfL

Posted Date: September 2nd, 2020

DOl: https://doi.org/10.21203/rs.3.rs-66815/v1

License: (c) (i) This work is licensed under a Creative Commons Attribution 4.0 International License. Read Full License 


\section{Abstract}

Background Paediatric Acute-onset Neuropsychiatric Syndrome (PANS) is characterized by an acute onset of obsessive compulsive disorder, combined with at least two other neuropsychiatric symptoms with similarly acute onset. Diagnostic criteria also require that no specific medical aetiology is identified. Although there are no verified aetiological biomarkers, PANS is assumed to be a neuroinflammatory disorder with a possible autoimmune aetiology. Neurochemical markers such as neurofilament light (NfL, a neuronal injury marker) and glial fibrillary acidic protein (GFAP, an astrocytic activation marker) have not been published for this patient group.

Methods Blood samples from 17 children meeting diagnostic criteria for PANS, after assessment at the Child Neuropsychiatry Centre, Sahlgrenska University Hospital, Gothenburg, Sweden, were analysed for serum concentrations of NfL and GFAP. Ten age-matched children without any neurological or psychiatric disorder served as a comparison group.

Results No difference was found in mean NfL and mean GFAP serum concentrations between children with PANS and controls. NfL and GFAP concentrations did not correlate with disease duration between PANS onset and time of blood tests.

Conclusion Neuronal injury and astrocyte activation do not seem to be a major event in PANS. The study group was small, and even if findings may be reassuring for parents and patients, they should be interpreted with caution and verified in larger cohort and possibly with other markers in both serum and CSF.

\section{Introduction}

The acronym PANS (Paediatric Acute-onset Neuropsychiatric Syndrome) is clinically defined by an abrupt, dramatic onset of obsessive-compulsive disorder or severely restricted food intake combined with at least two out of seven other similarly severe symptoms with acute onset; anxiety, emotional lability and/or depression, irritability/aggression and/or severely oppositional behaviours, behavioural (developmental) regression, deterioration in school performance (related to attention-deficit/hyperactivity disorder/ADHD-like symptoms, memory deficits, cognitive changes), sensory or motor abnormalities and somatic signs and symptoms, including sleep disturbances, enuresis, or urinary frequency. In addition, the research diagnostic criteria require that symptoms are not better explained by a known neurological or other medical disorder [1].

The term PANS was a modification of a preceding diagnostic term, PANDAS (Paediatric Autoimmune Neuropsychiatric Disorder Associated with Streptococcal infections), a similar clinical condition but implicating a temporal association with group A streptococcal infection and an ensuing autoimmune reaction [2]. By the term PANS aetiological associations were eliminated and hitherto no defined medical 
causes or reliable biomarkers have been identified, although infections and autoimmune reactions have been suggested [3]. Infections, mostly upper respiratory, are commonly reported symptoms preceding the onset of PANS [4]. In addition, autoimmune reactions, known to be associated with some neuropsychiatric disorders, have therefore also been assumed to have a connection with PANS [4]. Gilbert et al [5] emphasized the need to define better the clinical manifestations, laboratory and neuroimaging findings, therapeutic responses, and clinical course in these disorders and that, to date, there is no significant evidence supporting any particular aetiologies for PANDAS and PANS.

Neurofilament light chain $(\mathrm{NfL})$ protein, is a component of the neuron cytoskeleton and is released into the cerebrospinal fluid (CSF) and blood when neurons are injured, providing a neurodegenerative biomarker of neuronal damage [6]. An important aspect is that the NfL proteins indicate neuronal injury independent of causal pathways [6]. NfL levels in cerebrospinal fluid and in serum have been found to correlate significantly in paediatric patients with acquired demyelinating syndromes [7].

Glial fibrillary acidic protein (GFAP) is an astrocytic cytoskeletal protein, the expression and secretion of which is upregulated when astrocytes are activated [8]. In children with symptoms consistent with PANS, specific causes, such as N-methyl-D-aspartate receptor (NMDAR) encephalitis, the most common form of autoimmune encephalitis, needs to be considered [9]. Several studies have demonstrated that not only neurons, but also astrocytes express functional NMDA receptors and that the pathogenic antibodies against the NMDAR, causing the anti-NMDAR encephalitis, affect not only the neuronal receptors, but also the NMDAR on astrocytes [10].

In the 1990s, GFAP was analysed in cerebrospinal fluid (CSF) of children and adolescents with autism, of whom $70 \%$ also had intellectual disability (ID) and $21 \%$ had epilepsy, and results were contrasted with findings in similarly aged cases without autism or other neuropsychiatric disorders. GFAP levels in children with autism were found to be at a level almost three times higher than in the group of children without autism. In one aetiological subgroup of children with autism, consisting of three children with a basal ganglia disorder, the highest GFAP levels were found [11]. Also a more recent study, using serum levels of GFAP, demonstrated elevated levels in children with autism compared to controls [12].

To our knowledge it has not been systematically analysed whether children suffering from PANS would have detectable signs of neuronal/axonal or astrocyte cell damage. Since NfL and GFAP can now be analysed in serum, sampling in children is facilitated. Moreover, for both these biomarkers, serum concentrations have been shown to reflect the levels in the $\operatorname{CSF}[13,14,15]$. The aim of the present study was therefore to analyse concentrations of $\mathrm{NfL}$ and GFAP in serum of children meeting criteria for PANS.

\section{Methods}

Study participants

Our initial cohort of children with PANS consisted of 23 children, referred between 2015 and 2017 and assessed at the Child Neuropsychiatry Centre (CNC)/Gillberg Neuropsychiatry Centre in Gothenburg. 
During the study period, CNC was the referral center for children with suspected PANS in Gothenburg and the southwestern region of Sweden and these 23 children were deemed to meet clinical criteria for PANS. Data from the clinical assessments of these children have been reported previously [16].

For the present study 11 of the 23 children ( 7 boys), mean age 13 years, gave consent to have blood test taken for analysis of NfL and GFAP. In addition, 6 children ( 3 boys), mean age 9.6 years, later included in the cohort, were able to participate in blood sampling. Thus, 17 children could be analysed for serum NfL and GFAP.

The time from onset of PANS symptoms to the time point of blood sampling varied between 1 month and 5 years. At the time of assessment, however, all children had moderate to severe impairments that markedly influenced their school attendance and parental and family situation.

Four of the 17 patients had autism, no child in the study group had diagnosed intellectual disability. In addition to OCD and at least two other neuropsychiatric symptoms, four children had psychotic symptoms (delusions, hallucinations).

The comparison group included 10 children ( 5 boys, 5 girls), mean age 9.4 years, who did not have any neurological or psychiatric disorders. Their blood samples were taken in connection with investigation for non-neurological/non-psychiatric disorders, mainly allergic diseases, confirmed in their paediatric records, at a paediatric outpatient clinic in Gothenburg.

Serum analyses

$\mathrm{NfL}$ and GFAP were measured using ultrasensitive Single molecule array (Simoa) technology, using commercially available kits, according to instructions from the manufacturer (Quanterix, Billerica, MA). The analyses were performed in one round of experiments, using one batch of reagents by board-certified laboratory technicians who were blinded to clinical data. For a quality control (QC) sample with an NfL concentration of $15.0 \mathrm{pg} / \mathrm{mL}$, the repeatability was $7.2 \%$. For a QC sample with an NfL concentration of $47.3 \mathrm{pg} / \mathrm{mL}$, the repeatability was $1.7 \%$. For a QC sample with a GFAP concentration of $131 \mathrm{pg} / \mathrm{mL}$, the repeatability was $6.1 \%$. For a QC sample with a GFAP concentration of $365 \mathrm{pg} / \mathrm{mL}$, the repeatability was $4.3 \%$.

\section{Statistical analysis}

We conducted two-tailed t-tests in order to test mean differences between the PANS group and the comparison group. Since sample sizes were small, we cross-checked the t-test results with the nonparametric Mann-Whitney $U$ test, which yielded very similar p-values. We calculated Spearman correlations between the time point of PANS onset and the time point of blood sampling.

\section{Results}


Mean serum NfL was $4.7 \mathrm{pg} / \mathrm{ml}$ (SD 2.2) and $4.6 \mathrm{pg} / \mathrm{ml}$ (SD 1.7) in children with PANS and in the comparison group, respectively, t-test: $M_{\text {Diff }}=0.09,95 \% \mathrm{Cl}[-1.59,1.77], t(25)=0.11, p=.910$.

Mean serum GFAP was 103 pg/ml (SD 73.12) in the PANS group and 112 pg/ml (SD 64.37) in the comparison group, respectively, t-test: $\mathrm{M}_{\text {Diff }}=-9.75,95 \% \mathrm{Cl}[-67.28,47.78], \mathrm{t}(25)=-0.35, \mathrm{p}=.730$. MannWhitney U test gave similar results, $p=.941(\mathrm{NfL})$ and $p=.570$ (GFAP).

Time between onset of PANS symptoms and the time point of blood sampling did not relate to levels of either NfL $r=.14, p=.607$ or GFAP $r=-.20, p=.446$.

Mean serum GFAP of the four children with autism in the PANS group was $127.1 \mathrm{pg} / \mathrm{ml}$ (SD 121.3) and $95.8 \mathrm{pg} / \mathrm{ml}$ (SD 56.6) in the children with PANS without autism. No further calculations were performed due to very small numbers.

Table 1. Serum concentrations of Neurofilament light and Glial Fibrillary Acidic Protein Study groups

PANS group Control group

$n=17(10$ boys $) \mathrm{n}=10$ (5 boys)

mean age 11.3 years mean age 9.4 years

Mean (SD) Mean (SD) p-value

Neurofilament light $4.7 \mathrm{pg} / \mathrm{ml}(2.2) 4.6 \mathrm{pg} / \mathrm{ml}(1.7) \mathrm{p}=.910$

Glial fibrillary Acid Protein 103 pg/ml (73.12) 112 pg/ml (64.37) p = .730

\section{Discussion}

This study was based on 17 children referred to a child and adolescent neuropsychiatric clinic, at the time of the study the main referral center for children with suspected PANS in Gothenburg and the southwestern part of Sweden. All included children met the research criteria for PANS ${ }^{1}$ and had had an abrupt onset of OCD and at least two associated, severe neuropsychiatric symptoms. Co-occurring psychotic symptoms (delusions, hallucinations) were reported in $23.5 \%$ of the children in the PANS group, which is in line with other reports $[3,17]$. A basal ganglia involvement is presumed in PANDAS and there are studies implicating immune-mediated basal ganglia and striatal involvement also in PANS aetiology and in the pathogenesis of psychotic disorders [18]. However, as discussed by Gilbert et al [5] studies of PANDAS and PANS are inconclusive regarding pathophysiology, diagnostic markers and treatment. The need for cross-institutional collaboration and well-conducted investigations of underlying biological mechanisms in children with PANDAS/PANS has also been emphasized, given the severe nature of the symptoms and considerable effects on family and child functioning [19]. 
In the present study, we could not demonstrate increased levels of NfL and GFAP in serum of children with PANS, compared to a control group of children without symptoms of any neurological or psychiatric disorder. Levels of NfL and GFAP did not correlate with the elapsed time between onset of PANS and blood tests. In summary, our study did not reveal any signs of neuronal/axonal or astrocyte damage in children with PANS.

The four children with autism in our PANS group had GFAP concentrations that did not differ significantly from GFAP levels in the children with PANS without autism. However, the small study groups with only four children diagnosed with autism do not allow any conclusion to be drawn. These results differ from previous studies, in which elevated levels of GFAP were found in CSF [11] and serum [12] in children with autism compared to controls. However, none of the children with autism and PANS in our study had intellectual disability, while this was present in $70 \%$ of the children with autism reported in the 1990s [11].

Clear limitations of this study are the small study groups and the large differences in time periods between onset of PANS symptoms and blood sampling. However, all children had moderate to severe symptoms and impairment at blood sampling. A strength of the study is that all patients were clinically well defined and assessed by the same paediatricians, and that it was possible to use analyses of serum, since previous research has demonstrated correlation between these two biomarkers in cerebrospinal fluid and serum.

\section{Conclusions}

No indications of neuronal injury and astrocyte activation in PANS were found in this study. The study group was small, and even if findings may be reassuring for parents and patients, they should be interpreted with caution and verified in larger cohort and possibly with other markers in both serum and CSF.

\section{Declarations}

\section{Ethics approval and consent to participate}

The study was approved by the Gothenburg ethical review board. All parents and participants provided informed consent after receiving oral and written information of the study.

\section{Funding}

Swedish Brain Foundation. The funder of the study had no role in study design, data collection, data analysis, data interpretation, writing of the report, or the decision to submit for publication.

\section{Authors' contributions}

$\mathrm{KB}$ and $\mathrm{HZ}$ carried out the laboratory data analyses. All authors contributed to study design, data interpretation, drafting and revision of the manuscript. The corresponding author had full access to all the 
data in the study and had final responsibility for the decision to submit for publication.

\section{Consent for publication}

All authors gave final approval of the version to be submitted for publication.

Acknowledgements $\mathrm{HZ}$ is a Wallenberg Scholar supported by grants from the Swedish Research Council (\#2018-02532), the European Research Council (\#681712), Swedish State Support for Clinical Research (\#ALFGBG-720931), the Alzheimer Drug Discovery Foundation (ADDF), USA (\#201809-2016862), and the UK Dementia Research Institute at UCL.

KB is supported by the Swedish Research Council (\#2017-00915), the Alzheimer Drug Discovery Foundation (ADDF), USA (\#RDAPB-201809-2016615), the Swedish Alzheimer Foundation (\#AF-742881), Hjärnfonden, Sweden (\#FO2017-0243), the Swedish state under the agreement between the Swedish government and the County Councils, the ALF-agreement (\#ALFGBG-715986), and European Union Joint Program for Neurodegenerative Disorders (JPND2019-466-236).

We thank Lisa Dinkler and Martyna Galazka at Gillberg Neuropsychiatry Centre for statistical support.

Competing interests $\mathrm{HZ}$ has served at scientific advisory boards for Denali, Roche Diagnostics, Wave, Samumed and CogRx, has given lectures in symposia sponsored by Fujirebio, Alzecure and Biogen, and is a co-founder of Brain Biomarker Solutions in Gothenburg AB, a GU Ventures-based platform company at the University of Gothenburg, all unrelated to the work presented in this paper.

KB has served as a consultant or at advisory boards for Abcam, Axon, Biogen, Lilly, MagQu, Novartis and Roche Diagnostics, and is a co-founder of Brain Biomarker Solutions in Gothenburg AB, a GU Venturebased platform company at the University of Gothenburg, all unrelated to the work presented in this paper.

MJ has received research grants from Shire and been engaged as a speaker or consultant by Eli Lilly, Shire, Ginsana, PCM Scientific, Evolan, and New Nordic, all unrelated to the work presented in this paper.

\section{Availability of data and materials}

The datasets analysed during the present study are available from the corresponding author on request.

\section{References}

1. Swedo SE, Leckman JF, Rose NR. From research subgroup to clinical syndrome: Modifying the PANDAS criteria to describe PANS (pediatric acute-onset neuropsychiatric syndrome). Pediatr Therapeut. 2012;2:113.

2. Swedo SE, Leonard HL, Garvey M, Mittleman B, Allen AJ, Perlmutter S, et al. Pediatric autoimmune neuropsychiatric disorders associated with streptococcal infections: clinical description of the first 
50 cases. Am J Psychiatry. 1998;155:264-71.

3. Frankovich J, Thienemann M, Pearlstein J, Crable A, Brown K, Chang K. Multidisciplinary clinic dedicated to treating youth with pediatric acute-onset neuropsychiatric syndrome: presenting characteristics of the first 47 consecutive patients. J Child Adolesc Psychopharmacol. 2015;25:3847.

4. Chang K, Frankovich J, Cooperstock M, Cunningham MW, Latimer ME, Murphy TK, et al. PANS Collaborative Consortium. Clinical evaluation of youth with pediatric acute-onset neuropsychiatric syndrome (PANS): recommendations from the 2013 PANS Consensus Conference. J Child Adolesc Psychopharmacol. 2015;25:3-13.

5. Gilbert DL, Mink JW, Singer HS. A Pediatric Neurology Perspective on Pediatric Autoimmune Neuropsychiatric Disorder Associated with Streptococcal Infection and Pediatric Acute-Onset Neuropsychiatric Syndrome. J Pediatr. 2018;199:243-51.

6. Khalil M, Teunissen CE, Otto M, Piehl F, Sormani MP, Gattringer T, et al. Neurofilaments as promising biomarkers in neurological disorders. Nat Rev Neurol. 2018;14:577-89.

7. Wong YYM, Bruijstens AL, Barro C, Michalak Z, Melief MJ, Wierenga AF, et al. Serum neurofilament light chain in pediatric MS and other acquired demyelinating syndromes. Neurology. 2019;93:e96874.

8. Yang Z, Wang KK. Glial fibrillary acidic protein: from intermediate filament assembly and gliosis to neurobiomarker. Trends Neurosci. 2015;38:364-74.

9. Wang W, Zhang L, Chi XS, He L, Zhou D, Li JM. Psychiatric Symptoms of Patients With Anti-NMDA Receptor Encephalitis. Front Neurol. 2020;10:1330.

10. Ismail FS, Faustmann PM. Astrocytes and their potential role in anti-NMDA receptor encephalitis. Med Hypotheses. 2020;139:109612.

11. Ahlsén G, Rosengren L, Belfrage M, Palm A, Haglid K, Hamberger A, et al. Glial fibrillary acidic protein in the cerebrospinal fluid of children with autism and other neuropsychiatric disorders. Biol Psychiatry. 1993;33:734-43.

12. Wang J, Zou Q, Han R, Li Y, Wang Y. Serum levels of Glial fibrillary acidic protein in Chinese children with autism spectrum disorders. Int J Dev Neurosci. 2017;57:41-5.

13. Kuhle J, Barro C, Andreasson U, Derfuss T, Lindberg R, Sandelius Å, et al. Comparison of three analytical platforms for quantification of the neurofilament light chain in blood samples: ELISA, electrochemiluminescence immunoassay and Simoa. Clin Chem Lab Med. 2016;54:1655-61.

14. Novakova L, Zetterberg H, Sundstrom P, et al. Monitoring disease activity in multiple sclerosis using serum neurofilament light protein. Neurology. 2017;89:2230-7.

15. Högel H, Rissanen E, Barro C, Matilainen M, Nylund M, Kuhle J, et al. Serum glial fibrillary acidic protein correlates with multiple sclerosis disease severity. Mult Scler. 2020;26:210-9.

16. Johnson M, Fernell E, Preda I, Wallin L, Fasth A, Gillberg C, et al. Paediatric acute-onset neuropsychiatric syndrome in children and adolescents: an observational cohort study. Lancet Child Adolesc Health. 2019;3:175-80. 
17. Murphy TK, Patel PD, McGuire JF, Kennel A, Mutch PJ, Parker-Athill EC, et al. Characterization of the pediatric acute-onset neuropsychiatric syndrome phenotype. J Child Adolesc Psychopharmacol. 2015;25:14-25.

18. Silverman M, Frankovich J, Nguyen E, Leibold C, Yoon J. et al. Psychotic symptoms in youth with Pediatric Acute-onset Neuropsychiatric Syndrome (PANS) may reflect syndrome severity and heterogeneity. J Psychiatr Res. 2019;110:93-102., Mark Freeman G Jr.

19. Wilbur C, Bitnun A, Kronenberg S, Laxer RM, Levy DM, Logan WJ, et al. PANDAS/PANS in childhood: Controversies and evidence. Paediatr Child Health. 2019;24:85-91. 